http://dx.doi.org/ 10.22319/rmcp.v9i1.4345

Artículo

\title{
Función de producción de la ganadería de carne en la zona sur del Estado de México
}

\section{Function of beef cattle production in the South region of Mexico State}

José Luis Morales-Hernández ${ }^{1 *}$

Felipe de Jesús González-Razo ${ }^{1}$

Juvencio Hernández Martínez ${ }^{2}$

${ }^{1}$ Unidad Académica Profesional Tejupilco. UAEMEX. Rincón de Aguirre Tejupilco, CP 51400. Estado de México, México.

${ }^{2}$ Centro Universitario Texcoco, UAEMEX. Economía. Texcoco, México.

Autor de correspondencia: joseluistem@gmail.com

\section{- Resumen:}

Se estimó durante 2014-2015 una función de producción que relaciona la cantidad total de carne producida, a partir del pastizal natural, del total de animales y gasto de alimento; esto en 117 pequeñas y medianas empresas (Pymes) ganaderas de cuatro municipios del sur Estado de México, que sirvieron de base para estimar la función de producción Cobb Douglas, cuya información se analizó por mínimos cuadrados. Esta función de producción midió la eficiencia del recurso, en tres estratos: pequeños, medianos y grandes productores bajo el concepto de Pymes ganaderas. Así para las Pymes de los estratos I, con 2.6 a 26.5 ha y II, con 28 a 94 ha, al aumentar los factores de la producción en una proporción determinada, la producción crece menos que proporcionalmente los insumos. El estrato de Pymes III con 113 a 424 ha, mostró que al incrementar los factores en una determinada proporción la producción de carne crecerá a una mayor proporción. No se necesita aumentar la cantidad de alimento ni la superficie de pastizal para incrementar la cantidad total de carne, pero sí el número de animales. Los costos de oportunidad familiares y el precio de la carne son factores que otorgan ganancias al productor y le permiten mantenerse en el mercado de carne en el centro del país.

- Palabras clave: Cobb Douglas, Pymes, Costos, Administración, Registro contable. 


\begin{abstract}
- Abstract:
In 2014-2015 it was estimated a production function that relates the total quantity of meat produced from natural grassland, total animals and food spending. This was on 117 small and medium livestock business (Pymes, for Spanish acronym) in four municipalities of southern State of Mexico, which served as the base to estimate the Cobb Douglas production function, analyzed by mean squares. This study determined a production function to measure a resource efficiency, in three stratums: small, medium and large producers under the concept of the Pymes. For stratums: I, with 2.6 to 26.5 ha and II, with 28 to 94 ha, has to increase the factors of production in a certain proportion, the production grows less proportionately than inputs. Stratum III, 113 to 424 ha, showed that increasing factors in a certain proportion meat production would grow at a higher rate; economic indicators of meat production were calculated. Family opportunity costs and beef prices are factors that give profits to producers and allow staying in the meat market in the Central part of the country.
\end{abstract}

- Keywords: Cobb Douglas, Pymes, Costs, Administration, Accounting record.

Recibido 17/12/2016.

Aceptado 18/05/2017.

\title{
" Introducción N
}

La ganadería bovina en el Estado de México y en especial en los municipios de la parte sur de la entidad, constituye una actividad fundamental en el sector primario de la producción de carne, esto en virtud al número de cabezas de ganado. Estos municipios, durante 2014, contribuyeron con 104,840 cabezas de ganado, distribuidas de la siguiente manera: Tlatlaya 32,783 (31.3\%), Amatepec, 19,071 (18.2 \%), Luvianos 16,590 (15.8\%), Tejupilco 15,301 (14.6\%) y Temascaltepec y San Simón de Guerrero 21,095 (20.1\%); los cuales aportan el $20.3 \%$ del total de cabezas a nivel estatal, lo que representan un valor de la producción de 1'907,397 miles de pesos $^{(1)}$. Este tipo de ganado, es en su mayor parte dedicado a la producción de carne; únicamente el municipio de Tejupilco maneja producción de doble propósito leche-carne. 
En este trabajo se considera a las unidades de producción ganadera de carne como Pymes, ya que éstas, en México, constituyen el $97 \%$ del total de las empresas, generadoras de empleo del $79 \%$ de la población, y generan ingresos equivalentes al $23 \%$ del Producto Interno Bruto (PIB); lo anterior es una clara señal que debemos poner atención a este tipo de empresas y verlas como lo que en realidad son: la base de la economía mexicana ${ }^{(2,3)}$, por lo que, el análisis del proceso productivo de dichas empresas, se realizó en términos de eficiencia del recurso, pues de esto depende el grado de eficiencia económica ${ }^{(4)}$. Son por tanto las empresas las que generan utilidad o valor añadido, transformando los factores productivos en productos y distribuyéndolos para su consumo.

Existen metodologías que estiman la eficiencia del uso de recursos en empresas pecuarias para generar uno o más productos al final de su proceso productivo. Una de estas metodologías tiene como propósito la estimación de funciones de producción, mediante las cuales se establecen relaciones entre uno o más productos y los factores o insumos que intervienen en su producción, con lo cual se pueden predecir los valores de producción y determinar los niveles óptimos del uso de insumos y su productividad marginal ${ }^{(5)}$.

Algunos de los elementos de las funciones de producción utilizados en la ganadería bovina de carne son: el pastizal, la superficie ganadera empleada en la producción y la cantidad de alimento o suplemento empleado; estos se consideran los limitantes más comunes ${ }^{(4)}$.

Las funciones de producción, además, de permitir la determinación de la eficiencia biológica, permiten indagar en aspectos relacionados con la eficiencia económica, información que puede ser utilizada para propósitos de alimentación y selección ${ }^{(5)}$.

Con base en lo anterior, el objetivo del presente trabajo consistió en estimar una función de producción que describa la relación que guarda la producción total de carne, con tres factores que intervienen en el proceso productivo a través de la función Cobb-Douglas, y valorar los cambios productivos al modificar las cantidades de los recursos mencionados.

La hipótesis planteada fue que la producción de carne de bovino está en función directa de la cantidad de pastizal (PN), del número de cabezas de ganado (TA) y de la cantidad de alimento concentrado utilizado por la empresa (GA), es decir,

$\mathrm{Ho}(\mathrm{PTC})=\mathrm{PN}, \mathrm{TA}, \mathrm{GA}=0$

Los resultados permitirán detectar la eficiencia de los factores de la producción, y de esa manera obtener indicadores que permitan diseñar estrategias adecuadas para mejorar el proceso productivo de la ganadería bovina en esta región. Los resultados obtenidos pueden ser la base para evaluar las características específicas del crecimiento de la producción de carne en esta región, ya que permiten determinar el aporte de cada uno de los factores productivos a ese proceso. 


\section{" Material y métodos \|}

\section{- Ubicación del área}

La región sur del Estado de México está ubicada en el subtrópico mexicano, entre las coordenadas $18^{\circ} 21^{\prime}$ y $19^{\circ} 34^{\prime} \mathrm{N}$ y $99^{\circ} 16^{\prime}$ y $100^{\circ} 36^{\prime} \mathrm{O}$. Este espacio geográfico tiene límites con tres estados del país (Morelos, Guerrero y Michoacán) hacia el sur- sureste, limita con Morelos y Guerrero, al oeste tiene límites con Michoacán y parte de Guerrero ${ }^{(6)}$.

En el presente trabajo se consideraron los cuatro municipios más importantes en la producción ganadera que son Tlatlaya, Amatepec, Luvianos y Tejupilco, que contribuyen con el $100 \%$ (Cuadro 1), del total de cabezas del Distrito de Desarrollo Rural de Tejupilco ${ }^{(1)}$.

Cuadro 1. Municipios y muestra de productores de ganado de carne en el sur del Estado de México

\begin{tabular}{lll}
\hline Municipio & Muestra & \% \\
\hline Tejupilco & 14 & 12 \\
Luvianos & 21 & 18 \\
Amatepec & 50 & 43 \\
Tlatlaya & 32 & 27 \\
Total & 117 & 100 \\
\hline
\end{tabular}

Se realizó una clasificación de las Pymes ganaderas de carne en tres estratos de productores $^{(7,8)}$ : estrato I, con superficies entre 2.6 a 26.5 ha, estrato II con superficies de 28 a 94 ha y tercer estrato III con superficies de 113 a 424 ha. De esta manera, se consideraron 47 Pymes en el estrato I, 54 en el estrato II y 16 en el estrato III, para un total de 117, a utilizar en la función Cobb Douglas (Cuadro 2). 
Cuadro 2. Estructura de los estratos que integran las Pymes ganaderas del sur del Estado de México

\begin{tabular}{lcccc}
\hline Estrato & I & II & III & Total \\
\hline Superficie, ha & 2.5 a 26.5 & 28 a 94.5 & 113 a 424 & \\
Número Pymes & 47 & 54 & 16 & 117 \\
Porcentaje & 40 & 46 & 14 & 100 \\
\hline
\end{tabular}

La producción de carne por animal en cada estrato con suplementación ligera, alcanzó valores de 472, 452 y $402 \mathrm{~kg}$ durante el periodo de engorda. Las Pymes ganaderas de estos municipios tienen hatos constituidos con razas de Suizo Americano, Suizo Europeo, Simental, Cebú, Brahaman, Charolais y diversos cruzamientos entre éstas. El manejo del ganado de carne de estas unidades de producción tiene relación directa con el presupuesto que aplican los empresarios; en general, todos realizan pastoreo y suplementación complementaria.

\section{- Muestreo •}

La muestra de empresas se realizó en tres estratos ${ }^{(7,8)}$ basados en la superficie ganadera manejada, en la que se consideró a un total de 117 Pymes, distribuidas de la siguiente forma: Amatepec $43 \%$, Tlatlaya $27 \%$, Luvianos $18 \%$ y Tejupilco $12 \%$ (Cuadro 1).

Los datos obtenidos corresponden a un periodo de dos años (2014 y 2015), los cuales se registraron mediante un cuestionario en donde se recabó información sobre pastizal nativo (PN), total de animales (TA) y gastos de alimento (GA). Los datos se concentraron en una base de datos de Excel, donde se les dio orden, quedando como variable dependiente la producción total de carne (PTC) e independientes PN, TA y GA. A partir de esta base de datos se procedió a estimar la función de producción Cobb- Douglas ${ }^{(9)}$, lo cual permitió identificar el tipo de rendimientos asociados con el proceso productivo y estimar la elasticidad de los factores de la producción.

La función Cobb-Douglas es ampliamente utilizada para representar las relaciones entre un producto y las variaciones de los insumos tecnología, trabajo y capital ${ }^{(10)}$. Este modelo fue formulado por Knut Wicksell (1851-1926) y aplicado con la evidencia estadística por Charles Cobb y Paul Douglas en $1928^{(9)}$. El establecimiento de la función, parte de la observación 
empírica de la distribución de la producción entre L y K; la función de producción presenta la forma siguiente:

$$
Q=A L^{\propto} K^{\beta}
$$

\section{Donde:}

$\mathbf{Q}=$ producción total (el valor monetario de todos los bienes producidos durante un año);

$\mathbf{L}=$ insumo trabajo;

$\mathbf{K}=$ insumo capital;

A= factor total de productividad o Progreso Técnico (PTF);

$\boldsymbol{\alpha}$ у $\boldsymbol{\beta}$ son las elasticidades producto del trabajo y el capital, respectivamente. Estos valores son constantes determinadas por la tecnología disponible.

En dicha función, $\alpha$ y $\beta$ son los parámetros que representan el peso de los factores $\mathrm{K}$ y L (factores productivos) en la producción total. A es el progreso técnico o productividad total de los factores (PTF). La PTF no es una variable directamente observable, pues representa un estado no cuantificable formado por factores tales como: la organización empresarial, los conocimientos de los empresarios y trabajadores o el nivel de aplicación de tecnología. Por tanto, esta función de producción está compuesta por dos factores productivos que se mantienen constantes en el tiempo y un factor adicional (progreso técnico) ${ }^{(10)}$.

Para la estimación del modelo por Mínimos cuadrados ordinarios (MCO) se partió de una función lineal en los parámetros. Dado que la función de Cobb-Douglas no cumple esta condición, fue necesario realizar un proceso de linealización ${ }^{(10,11)}$. La transformación más común es tomar logaritmos en la función.

$$
Q=A L^{\propto} K^{\beta} e^{u}
$$

Aplicando logaritmos neperianos a la ecuación:

$$
\mathrm{Ln}(\mathrm{Q})=\ln (\mathrm{A})+\alpha \ln (\mathrm{L})+\beta \ln (\mathrm{K})+\mathrm{u}
$$

En la nueva forma funcional sobre la que se realizó la estimación del modelo, el parámetro $\alpha$ es la elasticidad producción-trabajo y $\beta$ es la elasticidad producción-capital. La elasticidad del producto midió la respuesta del producto a un cambio en los niveles del trabajo o del capital usados en la producción, si permanecen constantes los demás factores. Por ejemplo, si $\alpha=0.15$, un aumento del $1 \%$ en la cantidad de trabajo, provocaría un incremento aproximado del $0.15 \%$ en el volumen del producto.

Así, si: $\alpha+\beta=1$, la función de producción tiene rendimientos de escala constantes, es decir que si L y K aumentan cada uno el $20 \%$, Q aumenta también el $20 \%$. Esto significa que la 
función Cobb-Douglas es homogénea de grado 1, e implica que el costo mínimo es independiente del volumen de la producción, y depende sólo de los precios relativos de los factores de producción. Si $\alpha+\beta<1$, los rendimientos a escala son decrecientes, y si $\alpha+\beta>$ 1 los rendimientos a escala son crecientes.

En el presente trabajo, esta función se aplicó a la producción de carne de bovino, la cual quedó expresada como se muestra a continuación:

$$
\mathrm{PTC}=\beta_{0} P N_{1}^{\beta_{1}} T A_{2}^{\beta_{2}} G A_{3}^{\beta_{3}}+\mu
$$

\section{Donde:}

$\boldsymbol{\beta} \mathbf{o}=$ factor total de productividad;

$\boldsymbol{\beta 1}, \boldsymbol{\beta 2}, \boldsymbol{\beta 3}=$ elasticidades de PTC - PN, TA y GA;

PTC= producción total de carne;

$\mathbf{P N}=$ pastizal nativo;

$\mathbf{T A}=$ total de animales;

$\mathbf{G A}=$ gastos en alimentación; $\mu=$ error.

El análisis de varianza se sometió al criterio de aceptación de todas las variables con una $P<0.05$ y un coeficiente de determinación ajustado $\mathrm{R}^{2}$.

\section{Vesultados y discusión}

Para seleccionar en cada estrato de productores el modelo que mejor describe la función de producción, se consideraron los siguientes elementos: el valor ajustado del coeficiente de determinación $\left(\mathrm{R}^{2}\right)$, los resultados de la prueba de "F" y t de Student " $\mathrm{t}$ ".

Los resultados de cada estrato en cuanto a la función linealizada del tipo Cobb - Douglas, para el total de carne fueron los siguientes ${ }^{(9)}$ :

Estrato I

$$
\ln P T C_{1}=376.98-0.02335 \ln P N+0.95263 \ln T A+0.03979 \ln G A
$$




$$
\begin{array}{ccccc}
\text { (EE) } & (0.69320) & (0.02866) & (0.6811) & (0.08264) \\
\text { (t) } \quad(8.56) & (-0.81) & (13.99) & (0.48) \\
\mathrm{R} 2=0.8973 & \mathrm{Rj}=0.8892 \\
\sum\left(\beta_{1}+\beta_{2}+\beta_{3}+\beta_{4}\right)=-0.02335+0.95263+0.03979=>0,969 \text { Rendimientos } \\
\text { decrecientes }
\end{array}
$$

Estrato II

$$
\begin{aligned}
& \ln P T C_{2}=1218.43-0.0235 \ln P N+0.9456 \ln T A-0.0751 \ln G A \ldots . .(\mathrm{SII}) \\
& \begin{array}{lllll}
(\mathrm{EE}) & (0.34961) & (0.01750) & (0.03187) & (0.03373)
\end{array} \\
& \begin{array}{llll}
\text { ( }) \quad(20.32) \quad(-1.26) & (29.68)
\end{array} \\
& R 2=0.9467 \quad R j=0.9456 \\
& \Sigma\left(\beta_{2}+\beta_{3}+\beta_{4}\right)=(-0.0235+0.9456-0.0751)=0.847<1 \text { Rendimientos } \\
& \text { decrecientes }
\end{aligned}
$$

Estrato III

$$
\begin{gathered}
\ln P T C_{3}=629.23+0.00562 \ln P N+1.15982 \ln T A-0.12117 \ln G A \ldots \ldots \ldots .(\mathrm{SIII}) \\
\begin{array}{ccccc}
(\mathrm{EE}) & (1.0643) & (0.04139) & (0.10450) & (-0.10563) \\
(\mathrm{t}) & (606) & (0.14) & (11.09) & (-1.15) \\
\mathrm{R} 2 & =0.9124 & \mathrm{R} 2 \mathrm{j}=0.9001
\end{array} \\
\sum \beta_{2}+\beta_{3}+\beta_{4}=1.044>1 \text { Rendimientos crecientes. }
\end{gathered}
$$

Para el estrato I, la productividad marginal (PM) del PN indica que un aumento de una unidad de superficie en este insumo, provoca un decremento de $2.3 \%$ en la producción total de carne, cuando los otros dos factores (TA y GA) permanecen constantes. La PM del TA, muestra que el aumento de una unidad animal resultará en un aumento de la producción total de carne en $95.2 \%$, manteniendo constantes PN y GA. Para la PM de los gastos de alimentación, significa que el aumento de una unidad de este insumo, provoca un aumento de $3.9 \%$ de incremento en el total de carne, también manteniendo constantes los otros dos insumos (PN y TA).

En el caso del estrato II, la PM del PN indica que un aumento de una unidad de superficie provocará una disminución de $2.3 \%$ en la producción total de carne, permaneciendo constantes el total de animales y el gasto de alimento. La PM del TA revela que, un aumento de una unidad animal explica el aumento de $94.5 \%$ en la producción de carne, manteniendo 
constantes el pastizal natural y gasto de alimento. Similares resultados también en cuanto al número de animales son presentados por Pech, con un rango de ganancia de $987 \mathrm{~kg}$ de carne $^{(12)}$. La PM de GA, indica que un aumento de una unidad de este insumo producirá un aumento $7.5 \%$ de incremento en la producción de carne, igual manteniendo constantes pastizal natural y total de animales. Al contrario que Pech, el alimento en esta experiencia, produjo un aumento en la cantidad de carne ${ }^{(12,13)}$.

En el caso de las empresas de los estratos I y II, diversos autores sostienen que son los aprendizajes y la reducción de sus costos lo que les permite generar su economía de escala, y que poseen una más alta y variable tasa de crecimiento ${ }^{(13,14)}$; esto último lo logran con el apoyo de técnicos pecuarios regionales especializados, y el pastoreo les permite bajar el costo de la alimentación.

Por ultimo en el estrato III, la PM del PN indica que un aumento de una unidad de superficie provocó una disminución $5.6 \%$ en la producción total de carne cuando los dos insumos restantes total de animales y gasto de alimento permanecen constantes. La PM de TA muestra que un aumento de una unidad animal explica un incremento de $1.15 \%$ en producción total de carne, manteniendo constantes el pastizal natural y la cantidad de alimento. La PM de GA indicó que un aumento de una unidad de alimento provocó una disminución de $12.1 \%$ en la producción total de carne, también manteniendo constantes el pastizal natural y el total de animales.

Desde el punto de vista estadístico, en los tres estratos, los valores de la R2 fueron de 0.89 , 0.94, y 0.91, lo que significa que en las ecuaciones de regresión, la producción de carne se explica en una alta proporción por las variables indicadas, es decir, son pocos los factores no incluidos en la regresión que afecten de manera significativa el valor de la producción total de carne ${ }^{(12)}$.

\section{- Rendimientos a escala por sector •}

Sumando las elasticidades $\beta 1, \beta 2$, y $\beta 3$ de cada ecuación en cada sector, se obtiene la escala de rendimiento de la producción (Cuadro 3).

Cuadro 3. Resumen de las escalas de rendimiento en bovinos carne en el Distrito de Tejupilco 


\begin{tabular}{lccc}
\hline Estrato & Valor elasticidad & Escala & Clasificación escala \\
\hline I & 0.969 & $<1$ & Rendimiento escala decreciente \\
II & 0.847 & $<1$ & Rendimiento escala decreciente \\
III & 1.044 & $>1$ & Rendimiento escala creciente \\
\hline
\end{tabular}

En los estratos I y II, al aumentar los factores en una proporción determinada, la producción no crecerá más. En el tercer estrato, al aumentar los insumos en una proporción determinada, la producción de carne crecerá en una proporción mayor. El estrato III, de mayor superficie, resultó con economía de escala creciente, es decir, que si se aumenta en un determinado porcentaje alguno de los insumos, la producción aumentará en una proporción mayor. Los rendimientos crecientes en el estrato III, surgen de la especialización del capital y del trabajo, y tienden a reducir los costos de transacción, en este caso en la empresa ganadera. Esto se logró con mejoras genéticas del ganado y eficiencia en el manejo de la empresa ${ }^{(15)}$.

Otra opinión, cita que las empresas grandes (III), son eficientes porque operan bajo rendimientos crecientes, y pueden alcanzar economías de escala en amplios segmentos de su función de producción ${ }^{(16)}$. Se considera que los rendimientos crecientes se pueden obtener a través de los aprendizajes y de la acumulación de capital humano, y permitir una mejor asignación de recursos hacia el interior de la empresa, aún en condiciones informales ${ }^{(15)}$.

En un trabajo similar ${ }^{(17)}$ en la evaluación de factores de la producción en ganado de carne, se registró una elasticidad para pastoreo de 0.255 contra valores de los estratos I, II y III, -0.023 -0235 y 0.05 ; para el total de animales la elasticidad obtenida fue 0.573 , menor a la obtenida en los estratos de esta experiencia; para el alimento se reporta una elasticidad de 0.053. Ante los anteriores valores en el caso de pastoreo ${ }^{(18,19)}$, se recomienda el uso de pastoreo tecnificado, y cuando éste no cubra los requerimientos del ganado, se recomienda usar suplementos, situación que se da en la época de secas, por lo cual es recomendable usar bloques nutricionales, bloques de melaza o la utilización de pollinaza.

Una experiencia ganadera ${ }^{(17)}$, determinó la producción de carne en el sur de la provincia de Córdoba (Argentina), a través de funciones, tanto Cobb-Douglas como cuadráticas, utilizando como variables explicativas el porcentaje de pasturas, los gastos de suplementación y la carga animal, obteniendo un coeficiente de determinación ajustado mayor al $90 \%$ con niveles de significación de $P<0.05$.

En un proyecto más, los datos de 39 fincas revelan que la eficiencia técnica promedio fue de 0.7 , con $45 \%$ de los productores, presentando índices de eficiencia menor al promedio ${ }^{(18)}$. Resultados del modelo Logit indican que la productividad por animal, el tamaño de la finca, la carga animal, y la productividad de la mano de obra son las variables explicativas de las variaciones en eficiencia de este sistema. 
En un estudio de la eficiencia técnica del sector ganadero ${ }^{(20)}$ se detectó como factor primordial la alimentación como elemento primordial en productividad, y enseguida la salud del ganado, por ello se podría pensar en concentrar esfuerzos en el mejoramiento de los pastos, que es donde toman la mayoría de los nutrientes, haciendo énfasis en la aplicación de abonos, en particular orgánicos, trayendo consigo mayor productividad, lo cual se traduciría en mayores ingresos. Sirven para amortiguar los efectos de las sequías e inundaciones, impulsan la rentabilidad de la industria ganadera y ofrecen oportunidades de turismo y recreación que estimulan las economías locales.

La función de producción describe la combinación de factores, total de animales, total de pastizal o total de alimento que permiten obtener un mismo nivel de producto, en este caso total de carne, lo cual permite diferenciar entre tecnologías intensivas en número de animales y total de pastizal o de alimentos.

Respecto al valor de la elasticidad para el total de animales, los valores de diferentes experiencias $^{(12,16)}$ indican que la cantidad de animales es la adecuada, y aún puede incrementarse su número, para mantener la producción de carne que demanda el mercado estatal.

\section{Conclusiones e implicaciones}

Los estratos I y II indican que los empresarios con menor superficie ganadera y los empresarios de mediana superficie, en los que los rendimientos a escala son decrecientes, o sea, ante una expansión proporcional igual en todos los insumos, el producto se incrementa en una proporción menor, donde se cumple el postulado de rendimientos marginales decrecientes en los factores. Los productores del estrato III de mayor superficie están obteniendo rendimientos crecientes a escala, es decir al aumentar los insumos en una determinada proporción la producción aumenta en mayor proporción a los mismos. La hipótesis para la función de producción se cumple sólo para el estrato III, produce rendimientos crecientes a escala. Algunos de los factores limitantes de la producción bovina de carne son el número de animales y la cantidad de alimento proporcionada en cada empresa. Aunado a lo anterior, en los últimos meses, la elevación del precio de la carne es un factor que más ha incentivado al productor y aumentar su ganancia, situación que ha sido provocada por factores climáticos en la parte norte y noreste del país. 


\section{- Literatura Citada}

1. SAGARPA. Secretaria de Agricultura Ganadería Desarrollo Rural Pesca y Alimentación. Distrito de Tejupilco. Departamento de Estadística. 2014.

2. Álvarez J. Pymes de México sin Planeación de Negocios. El Economista.2014.http:// eleconomista.com.mx/industrias/2013/01/25/pymes-mexico-sin-planeacion-negocios. Consultado 10 Dic, 2014.

3. COEPES. Comisión Estatal Para la Planeación de la Educación Superior. Importancia de la Pymes en México. Guanajuato. file:///H:/COBDUG/ IMPORTANCIA\% 20DE\%20LAS\%20PYMES\%20EN\%20MÉXICO. 2015. Consultado 12 Feb, 2016.

4. Debertin DL. Agricultural production economics. New York: MacMillan; 1982.

5. Ramírez AN, Mungaray LA, Ramírez UM, Texis FM. Economías de escala y rendimientos crecientes. Economía Mexicana 2010; XIX(2):213-230.

6. Franco MS, Cadena RI, González TA, Orozco HE, Salazar BS, Coronado RJ, et al. Atlas del Estado de México. Gobierno del Estado de México. Universidad Autónoma del Estado de México. Toluca, México. 1992.

7. Hernández SR, Fernández C, Baptista DF. Metodología de la Investigación. $3^{\mathrm{a}}$ ed. México: Mc Graw-Hill Interamericana; 2014.

8. Vilez ED. Estadística básica para universitarios. EUNSA Navarra 2001.

9. Cobb CW, Douglas PH. A theory of production. Am Econom Rev 1928;18:139-165.

10. Gujarati ND, Porter DC. Econometría. México. 5a ed. México, DF: Mc Graw Hill Interamericana; 2014.

11. Aguirre VL, Venezian E. Estimación de funciones de producción para la zona rural vecina a Chapingo. Agrociencia 1975;20:13-19.

12. Pech MV, Santos FJ, Montes PR. Función de producción de la ganadería de doble propósito de la zona oriente del estado de Yucatán, México. Téc Pecu Méx 2002;40(2):187-192.

13. Pyke F, Becattini G, Sengerberger W. Industrial Districts and Inter-firm Cooperation in Italy, Génova, International Intitute for Labour Studies. 1990.

14. Loverman G, Senserbeger W. The re-emergence of small-scale production: An international comparison. Small Business Econom 1991;(3):1-37. 
15. Krugman P. Urban concentration: The role of increasing returns, and transport cost. Ann Regional Sci Rev 1996;(19):5-30.

16. Pariani AO, Martínez G, Antón R, Peinado MJ. Optimización de producciones complementarias y competitivas en el Noroeste de la provincia de la pampa. Cienc Vet 2004;6(1):70-90.

17. Ruiz, D. Modelos avanzado de gestión y optimización de la producción bovina en la región de la pampa húmeda Argentina [tesis doctoral]. España: Universidad de Córdoba; 1997.

18. Ortega, SL, Albornoz GA, Segovia LE. Índice de productividad total de la ganadería de doble propósito del municipio Cólon, estado de Zulia-Venezuela. Rev Cient /Maracaibo 2007; 17(3):268-274.

19. FIRA. Fideicomisos Instituidos en Relación con la Agricultura (FIRA) Comportamiento de Ganado de doble propósito en el trópico. Mérida Yucatán. Banco de México. 1966.

20. CCA. Comisión Cooperación Ambiental. Industria ganadera, comercialización de ganado de engorda y pastizales de América del Norte: situación y tendencias. Montreal, Canadá. 2015. 